\title{
Pursuit Eye Movements Involve a Covert Motor Plan for Manual Tracking
}

\author{
Claudio Maioli, Luca Falciati, and Tiziana Gianesini \\ Department of Biomedical Sciences and Biotechnologies, University of Brescia, 25123 Brescia, Italy
}

\begin{abstract}
When we make an aiming movement toward a moving visual object, eye- hand coupling is of paramount importance for accurate motor performance. Some studies have suggested that both gaze and manual tracking control systems are driven by a common command signal. However, it has never been demonstrated that a motor plan for the arm is produced even when the object is tracked by the eyes alone. By applying transcranial magnetic stimulation to the motor cortex, we show for the first time that ocular tracking is linked to an overall decrease in the excitability of the motor control system of the relaxed upper limb, as estimated from the amplitude of the motor evoked potentials recorded in contralateral hand and wrist muscles. Furthermore, this reduced excitability is modulated in a manner compatible with a subthreshold neural activation encoding a manual tracking response to the same target pursued by the eyes. In addition, excitability changes are contingent on upper-limb posture, because they are present only with a pronated forearm and not with a supinated hand position. We provide direct evidence that, if the arm is held in a congruent postural configuration, tracking a moving object always entails a coordinated motor plan, which involves both gaze and hand movements. Active inhibitory mechanisms are activated to prevent an overt arm movement, whenever a manual tracking is not requested. Our data provide strong evidence in favor of the existence of a common drive to both eye and hand tracking systems.
\end{abstract}

Key words: smooth pursuit; manual tracking; eye-hand coupling; motor excitability; transcranial magnetic stimulation; motor evoked potentials

\section{Introduction}

Pointing and tracking objects under visual guidance are some of the most common motor behaviors in everyday life. In these activities, arm movements are typically associated with eye movements toward the same goal. Of course, eyes and hand can move alone, but converging evidence from a large number of studies indicates that performance considerably improves when they move together (Prablanc et al., 1979; Mather and Lackner, 1980; Vercher et al., 1994; Neggers and Bekkering, 1999; van Donkelaar and Staub, 2000; Engel and Soechting, 2003). In particular, smooth pursuit (SP) eye movements become more accurate and contain fewer catch-up saccades when they are accompanied by an arm tracking movement (Koken and Erkelens, 1992). The reverse is also true, as manual tracking performance is more precise if eye and hand follow the same spatial trajectory, than when tracking is made by the hand alone (Miall and Reckess, 2002). To account for these findings, a mutual coupling between eye and hand motor control systems has been proposed, in which a performance improvement results from an exchange of nonvisual signals between separate controls (Gauthier et al., 1988; Lazzari et al., 1997; Scarchilli and Vercher, 1999).

However, there are data also suggesting that at least part of the central controller could be in common to both arm and oculo-

\footnotetext{
Received Feb. 13, 2007; accepted May 19, 2007.

Correspondence should be addressed to Prof. Claudio Maioli, Dipartimento di Scienze Biomediche e Biotecnologie, Università di Brescia, Viale Europa 11, 25123 Brescia, Italy. E-mail: maioli@med.unibs.it. DOI:10.1523/JNEUROSCI.1832-07.2007

Copyright $\odot 2007$ Society for Neuroscience $\quad$ 0270-6474/07/277168-06\$15.00/0
}

motor systems. If the trajectory of a moving target presents an abrupt change in direction, eye and hand tracking movements show striking similarities in kinematics despite the considerable inertial differences of the two systems (Engel et al., 2000). Furthermore, by using adaptation to increase SP gain, an enhancement in amplitude is also concomitantly observed in manual tracking responses, even when eye motion is restricted (van Donkelaar et al., 1994). To explain these observations, it has been proposed that a common command signal is driving both ocular and manual tracking responses and that the two systems share at least partially a common neural controller. However, no direct evidence for this hypothesis has been so far provided.

In the presence of a common drive, we would expect that a motor plan for manual tracking is formed even when subjects are pursuing a moving object by the eyes alone. Transcranial magnetic stimulation (TMS) is a tool that allows us to disclose an ongoing motion planning by dynamically measuring the excitability changes of the corticospinal system (CSS) in behaving subjects, as estimated from motor evoked potential (MEP) amplitude in the relaxed muscles. For instance, TMS has been successful in demonstrating that the observation of others' actions (Fadiga et al., 1995, 2005) or the mental simulation of a movement (Izumi et al., 1995; Kasai et al., 1997; Hashimoto and Rothwell, 1999) induces excitability changes in the CSS, which are coherent with the observed or imagined action. The aim of this study is to ascertain whether ocular tracking of a moving object inherently involves changes of excitability in the CSS of the resting arm and to verify whether the observed responses are com- 


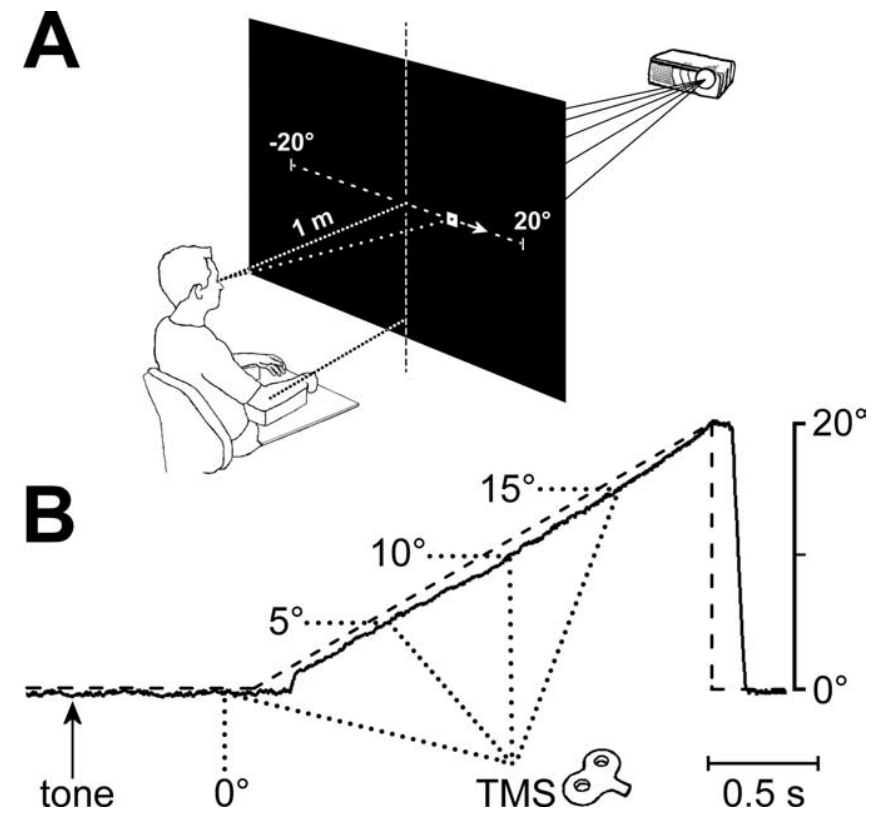

Figure 1. Experimental setup and protocol. $\boldsymbol{A}$, White visual stimuli were rear-projected on a wide tangent black screen placed $1 \mathrm{~m}$ in front of the subject. The head was immobilized by using a chin rest and a head-support device (not shown). Notice the arm- hand posture (in this figure pronated) adopted by the subject with respect to the central vertical meridian. $B, E O G$ recording (solid line) during SP eye movement, at a constant target velocity of $10 \%$ (dashed line). A warning tone was delivered $1000 \mathrm{~ms}$ before onset of the target movement. TMS pulses were randomly delivered either $100 \mathrm{~ms}$ before SP onset or during the tracking eye movement, at gaze eccentricities of $5^{\circ}, 10^{\circ}$, or $15^{\circ}$.

patible with a motor plan for an aiming movement of the hand toward the gaze target.

\section{Materials and Methods}

Subjects. Fifty-nine adult volunteers (22 male and 37 female; mean age, 21.9 years; range, $18-34$ ) with no history of head trauma or neurological disease participated in the study. All subjects were right handed (as measured by the Edinburgh handedness inventory) and naive to the purpose of the experiment. This study was conducted in accordance with the ethical guidelines set forth by the Declaration of Helsinki. Written informed consent was obtained from all participants.

Experimental protocol. Subjects were sitting comfortably, with their forearms resting relaxed on a horizontal support pointing toward the central vertical meridian and with the hands slightly hanging downward as a result of gravity (Fig. 1A). Two experimental series were performed with identical visual and TMS stimulation protocols, but differing as far as the static pronated/supinated posture of the forearm adopted by the subject. Thirty-four subjects participated in the series of experiments with pronated forearm, and 35 subjects participated in the series with the supinated forearm (10 subjects were recruited in both series). Visual stimuli were rear-projected on a wide tangent black screen $(160 \mathrm{~cm}$ in width and $120 \mathrm{~cm}$ in height) placed $1 \mathrm{~m}$ in front of the subject. Participants were instructed to fixate for $4 \mathrm{~s}$ a white central cross. One second after a warning tone, the fixation cross turned to a white square (subtending $0.6^{\circ}$ of visual angle) and started moving at a constant velocity of $10 \%$ along the horizontal meridian, up to a $20^{\circ}$ eccentric position. Subjects had to track the target by the eyes alone as accurately as possible. During each trial (Fig. $1 \mathrm{~B}$ ), single-pulse TMS was randomly delivered either 100 $\mathrm{ms}$ before target motion or during the SP eye movement, triggered by the electrooculography (EOG) signal at gaze eccentricities of $5^{\circ}, 10^{\circ}$, or $15^{\circ}$. Each experimental session comprised an overall number of 140 trials ( 20 trials for each of the seven eye eccentricity conditions). Leftward and rightward SP directions as well as occurrences of TMS pulses during central fixation or at different eye positions were randomly intermixed, so that subjects were unable to predict timing and gaze eccentricity of TMS.
Eye movement and electromyogram recording. Horizontal and vertical eye movements were recorded (cutoff $0-200 \mathrm{~Hz}$ ) by means of DC-EOG. $\mathrm{Ag}-\mathrm{AgCl}$ electrodes were placed at the external canthi and above and below the right eye. EOG calibration was frequently repeated during the experimental session, and drift of DC offset was compensated within each trial by making the subject look at a central fixation cross before onset of target motion. The subject's head was stabilized using a combination chin rest and head-support device. Surface electromyograms (EMGs) were recorded on the right-hand side from first dorsal interosseous (FDI), abductor digiti minimi (ADM), and extensor carpi radialis (ECR) muscles. EMG signals were amplified $1000 \times$ with a bandwidth from $0.2 \mathrm{~Hz}$ to $1 \mathrm{kHz}$. Furthermore, attention was paid, as a criterion of trial acceptance, that subjects were keeping their muscles completely relaxed, in the absence of any detectable EMG activity for the entire duration of the tracking task.

EOG and EMG signals were digitally converted at a sample rate of 4 kHz (PCI-MIO-16E-4; National Instruments, Austin, TX) and analyzed off-line by means of custom-written Labview software (National Instruments).

Transcranial magnetic stimulation. A $70 \mathrm{~mm}$ figure-of-eight double coil connected to a MagStim Super Rapid magnetic stimulator (Mag1450-00; MagStim, Whitland, UK) was positioned over the left motor cortex, contralaterally to the EMG-recorded muscles. The coil was placed tangentially to the scalp with the handle pointing backward and laterally at a $45^{\circ}$ angle to the sagittal plane, inducing a posterior-anterior current in the brain. This orientation was chosen because the lowest motor threshold is achieved when the induced electrical current in the brain flows approximately perpendicular to the central sulcus (Mills and Nithi, 1997).

The scalp site at which MEPs were elicited in FDI muscle at the lowest stimulus strength was determined. Once optimal scalp site was found, coil was securely fixed in place by means of an apt mechanical device. The response threshold was defined as the stimulus intensity at which five of 10 consecutive stimuli at the optimal site evoked an MEP with a peak-topeak amplitude of at least $100 \mu \mathrm{V}$ in the relaxed muscle. Stimulus intensity during the entire stimulation paradigm was set at 1.2 times the FDI motor threshold. The amplitudes of TMS pulses applied to the motor cortex turned out to be nonsignificantly different between "pronated" and "supinated" experimental series, being 70.6 and $69.2 \%$, respectively, of the maximum power of the magnetic stimulator. This stimulation intensity at the optimal scalp site for FDI also evoked MEPs in ADM and ECR muscles in all experimental sessions, although generally with a considerably lower amplitude. Low-amplitude responses could introduce a methodological bias in the presence of SP-induced inhibitory effects. In fact, the assessment of a reduction in CSS excitability would be certainly masked if control MEP amplitude were measured with stimulation intensities at or just above threshold. Therefore, for each muscle, subjects were included in the analysis only if the mean peak-to-peak MEP amplitude during central fixation was $>140 \mu \mathrm{V}$, to insure that excitability changes could be measured against a reliable baseline. In fact, we observed that only with control amplitudes above this baseline level did TMS induce recordable MEPs in the large majority of trials, even in the presence of a reduction of CSS excitability during SP eye movements (see Results). In the experimental series with pronated forearm, this acceptance criterion was fulfilled in 34, 23, and 27 subjects for FDI, ADM, and ECR muscles, respectively. For the same muscles, the subjects included in the analysis in the experiments with a supinated forearm posture were 29, 27 , and 28 , respectively.

Statistical analysis. MEP amplitudes are continuous variates characterized by a very large variability among subjects in the mean and SD of their statistical distribution. In particular, data from each subject tended to have similar coefficients of variation, as SD generally increased as a function of the sample mean. Therefore, to pool together data from all subjects and apply statistical significance tests, some kind of data transformation is required. An appropriate transformation, in common use in statistics, is the standardized transform defined as follows: $X=(x-$ $\mu) / \sigma$, where $X$ is the standardized variable, and $x$ is the original variable having a distribution with $\mu$ mean and $\sigma \mathrm{SD}$. By this transformation, MEP amplitude becomes a standard measure with zero mean and unitary SD. Therefore, this transformation was separately applied for each sub- 
ject, to normalize experimental variability and permit a direct data comparison.

We can expect that changes in gaze direction do not affect in a significant manner the intrinsic variability of MEP amplitude. Thus, it is reasonable to assume that the observed effects mainly reflect a shift of the mean MEP amplitude, with negligible changes in the variance for a given gaze position (residual variance). Therefore, for the computation of the standardized MEP amplitude, we chose to use the residual variance as a measure of variability within each subject. By considering measurements of MEP amplitude at the different gaze positions as independent samples, we can write $S_{T}=S_{B}+$ $S_{W}$, where $S_{T}$ is the total population variance:

$$
S_{T}=\frac{\sum_{1}^{N} x^{2}-\left(\sum_{1}^{N} x\right)^{2} / N}{N-1}
$$

( $N$ is the number of data points); $S_{B}$ is the variance between samples:

$$
S_{B}=\frac{\sum_{1}^{n} N_{\mathrm{i}}\left(\bar{x}_{\mathrm{i}}-\bar{x}\right)^{2}}{n-1}
$$

( $n$ is the number of samples); and $S_{W}$ is the variance within samples or residual variance: $S_{W}=S_{T}-S_{B}$. Because our aim was to test the effect of gaze on mean MEP amplitude, standardized variables were computed by using the square root of the residual variance $S_{W}$, in order not to cancel the data variability induced in each subject by changes in gaze position.

Finally, standardized MEP amplitudes can be easily inverse transformed to the original variable as follows: $x=\sigma X+\mu$. Therefore, for an approximate evaluation of the mean changes in MEP amplitude expressed in microvolts, induced by gaze direction in the overall population, standardized values can be inverse transformed by using the average values of mean and variance, computed across all experimental subjects. After this transformation, the changes in MEP amplitude $(\triangle M E P \%)$ between the responses recorded during leftward $\left(M E P_{\text {left }}\right)$ and rightward $\left(M E P_{\text {right }}\right)$ gaze directions, can be computed as the percentage variation of their respective average values $\left(M E P_{\text {mean }}\right)$ through the following formula: $\triangle M E P \%=\left(M E P_{\text {left }}-M E P_{\text {right }}\right) / M E P_{\text {mean }} \times 100$.

\section{Results}

\section{Ocular tracking performed with the forearm held in a pronated posture}

In a first series of experiments, subjects were asked to keep their forearm in a pronated, relaxed posture. As expected, peak-topeak MEP amplitudes are characterized by a very large variability among subjects in the mean and SD. Therefore, to pool together data from all subjects and apply statistical significance tests, raw data are transformed in standardized variables, by subtracting within each subject the average MEP amplitude measured before SP onset and normalizing by the square root of the subject's residual variance (i.e., the variance within groups defined by gaze eccentricity) (see Materials and Methods, Experimental protocol). As a result, MEP amplitudes are represented as the difference from the mean value recorded during the fixation of a stationary target, and MEP changes are expressed in all subjects as a fraction of the experimental variability of the data.

The analysis of the pooled data neatly demonstrate that SP eye movements considerably modulate the excitability of CSS of all three investigated muscles. Figure $2 A-C$ illustrates the average values across subjects of standardized MEP amplitudes as a function of gaze eccentricity, with negative eye position values indicating a leftward direction. Empty symbols represent the mean reference values measured during fixation of a stationary central point. Filled symbols indicate that MEPs were recorded during SP. It can be readily seen that the execution of an eye tracking movement induces a clear decrease in excitability of the CSS for all muscles, revealed by a very significant reduction of the mean MEP amplitude measured during SP with respect to fixation. Figure $2 D$ shows the average values for leftward and rightward SP in all muscles. In all cases, the statistical comparisons ( $t$ test) of mean MEP amplitudes indicate a highly significant difference $(p<0.01)$ between SP and fixation samples. Furthermore, ANOVA demonstrates that the decrease in standardized MEP amplitude does not show any statistically significant difference among muscles $(F=2.478 ; p=0.084)$.

Based on these results, it is possible to conclude that fixation of a stationary target and tracking a moving object by the eyes alone represent two very different conditions as far as the overall level of excitability of the CSS controlling the hand. Therefore, to study the effects of gaze eccentricity and eye movement direction on MEP amplitude, the analysis was restricted to SP data by excluding the responses recorded during central fixation. A two-way ANOVA (Table 1) was performed on the pooled standardized data with gaze eccentricity and SP direction as grouping factors. The analysis shows that in all muscles, MEP amplitude is independent of gaze eccentricity but is affected in a highly significant manner by SP direction (side factor). As it can be better appreciated in Figure $2 D$, responses in FDI and ECR are larger during leftward eye tracking, whereas the opposite occurs in ADM. It should also be noted that in all muscles, no statistically significant interactions were found between side and eccentricity factors.

We would also like to point out that from a statistical standpoint, all described effects are very robust. In fact, despite the very 
Table 1. Two-way ANOVA for standardized MEP amplitude

\begin{tabular}{|c|c|c|c|c|c|}
\hline \multirow[b]{2}{*}{ Muscle (subjects) } & \multirow[b]{2}{*}{$N$} & \multicolumn{2}{|c|}{ Eccentricity } & \multicolumn{2}{|l|}{ Side } \\
\hline & & $F_{(2, N-6)}$ & $p$ & $F_{(1, N-6)}$ & $p$ \\
\hline \multicolumn{6}{|l|}{ Pronated forearm } \\
\hline FDI (34) & 3688 & 0.328 & 0.720 & 5.990 & $0.014^{* *}$ \\
\hline $\operatorname{ADM}(23)$ & 2527 & 1.788 & 0.167 & 5.664 & $0.017^{* *}$ \\
\hline $\mathrm{ECR}(27)$ & 2934 & 2.305 & 0.100 & 6.035 & $0.014^{* *}$ \\
\hline \multicolumn{6}{|l|}{ Supinated forearm } \\
\hline FDI (29) & 3175 & 0.359 & 0.699 & 0.501 & 0.479 \\
\hline $\operatorname{ADM}(27)$ & 2860 & 0.599 & 0.549 & 0.095 & 0.757 \\
\hline $\mathrm{ECR}(28)$ & 3091 & 0.318 & 0.727 & 0.148 & 0.700 \\
\hline
\end{tabular}

Two-way ANOVA of standardized MEP amplitudes with respect to SP direction (side) and eccentricity in both forearm posture conditions. $N$ is the total number of trials. Asterisks denote statistical significance. For all muscles, side $X$ eccentricity interaction is not significant $(p>0.15)$.

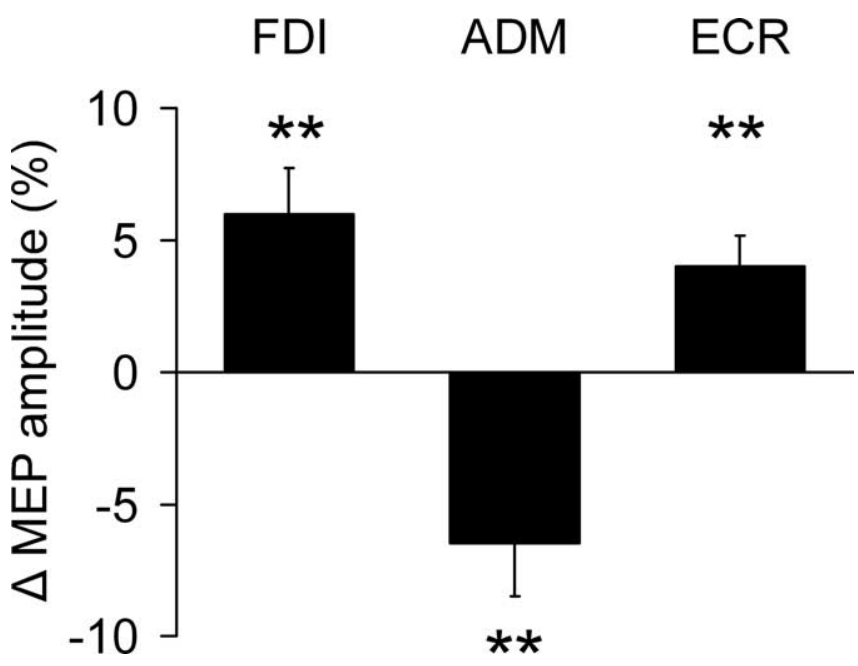

Figure 3. Percentage variation of MEP amplitudes. Percentages are expressed with respect to the population mean of the average modulation in MEP amplitude between leftward and rightward SP directions (see computation formula in Materials and Methods). Error bars represent the SEMs. Asterisks indicate a statistically significant difference from zero.

large variation among subjects in the SDs of MEP responses, the same ANOVA analysis applied to the raw data (without the transformation to standardized variables) yields identical results as far as the assessment of statistically significant differences between experimental conditions.

To evaluate mean MEP changes in more meaningful units, standardized values were inverse transformed to microvolts, by using the mean and SD of the original experimental data (see Materials and Methods). Thereafter, in each muscle the mean change in MEP amplitude was computed as percentage variation of the average control value of the overall population. The mean absolute MEP amplitudes across subjects during central fixation were $1358 \pm 1660,502 \pm 505$, and $383 \pm 297 \mu \mathrm{V}$, for FDI, ADM, and ECR muscles, respectively. The population average decrease in MEP amplitude during SP eye movements was 10.7, 13.7, and $7.8 \%$ of fixation values, respectively. Finally, the percentage differences in MEP size between leftward and rightward SP were similar (Fig. 3), although of opposite sign, in FDI and ADM muscles (5.97 and $-6.47 \%$ of the mean values during fixation, respectively). In contrast, only a smaller variation of $4.00 \%$ was observed in ECR.

\section{Ocular tracking performed with the forearm held in a supinated posture}

Our working hypothesis, to interpret the so far described data, was that the observed changes in excitability of the CSS control- ling hand movements unveil the existence of a subthreshold motor plan associated with SP eye movements. If so, we can expect that a different arm posture would dramatically modify the pattern of MEP modulation recorded in the various muscles. To test this prediction, we made another series of experiments with identical visual and TMS stimulation protocols but asking the subjects to keep their forearm relaxed in a supinated posture (palm up). As a result, all fingers were held in a semiflexed position, and the wrist was slightly extended by gravity. As in the "pronated" experimental series, the forearm was resting on a horizontal support pointing toward the central vertical meridian. Most subjects felt that this posture was somewhat unnatural but managed anyhow to carry on the task without difficulties.

The average absolute MEP amplitudes across subjects during central fixation with a supinated forearm were $951 \pm 1029,632 \pm$ 642 , and $337 \pm 227 \mu \mathrm{V}$ for FDI, ADM, and ECR muscles, respectively. These mean MEP amplitudes were not significantly different from those measured with a pronated posture of the forearm ( $t$ test; $p>0.1)$. Notice that also the average threshold intensity of magnetic stimulation to elicit MEPs in FDI muscle was not significantly different in the two experimental series (see Materials and Methods). Therefore, it is possible to conclude that, in our conditions, the baseline (central fixation) excitability of upperlimb CSS was not affected by the adopted forearm posture.

Figure 4 summarizes the effects of the new arm posture on MEP amplitude. It can be clearly seen that SP does not induce any decrease in excitability of the arm CSS, because mean MEP amplitudes measured during ocular tracking to both leftward and rightward directions (Fig. $4 D$ ) do not differ significantly ( $t$ test) from fixation values. Indeed, a tendency toward a reduction in MEP amplitude can be observed in FDI muscle, but also in this case the difference is not statistically significant $\left(_{(3776)}=1.385\right.$; $p=0.166)$. Furthermore, two-way ANOVA demonstrates that neither SP direction nor eye eccentricity during ocular tracking affect MEP amplitude in a significant manner (Table 1). We must then conclude that SP-induced changes of excitability in the CSS are contingent on the adopted posture of the arm during the tracking task.

\section{Discussion}

In this study, we demonstrate for the first time that tracking a moving object with the eyes inherently involves excitability changes in the motor control system of the arm, in the absence of any overt limb movement or sign of EMG activation. Changes in excitability consist in an overall inhibition of the upper-limb CSS, which is modulated in a highly specific manner in the different muscles, depending on the direction of SP eye movements. This cannot be considered a generic effect attributable to the arousal level or to a generalized variation of cortical excitability bound to task execution. In fact, the pattern of changes in MEP amplitude varies among muscles and is contingent on the adopted arm posture.

In the following, we argue that the observed changes in CSS excitability reveal that a subthreshold motor plan for manual tracking is formed in association with SP eye movements, suggesting the existence of a common drive to ocular and manual tracking systems. Because the task requires that the target be tracked by the eyes alone, the arm motor program is accompanied by an active inhibition of the upper-limb motor system, to decrease the risk of an overt movement generation. 


\section{MEP modulation during SP unveils a motor plan for manual tracking}

When subjects perform SP eye movements with a pronated posture of the forearm, there is a high degree of congruence between the modulation of MEP amplitude and pursuit direction. For instance, FDI (abductor of the index finger) on the righthand side shows a higher excitability during leftward than during rightward SP eye movements. Accordingly, the activation of this muscle would induce a leftward movement of the index finger. In contrast, MEP amplitudes in ADM, whose activation would induce a rightward deviation of the little finger, become larger when eyes are pursuing a rightward moving target.

As for the ECR, this muscle is not a pure extensor of the wrist, but its action is equally strong in producing also a radial deviation of the hand (Bawa et al., 2000). Therefore, a contraction of the ECR on the right side with a pronated forearm (Fig. 1) would produce an upward and leftward movement of the hand. Accordingly, in our data MEP amplitude in ECR are significantly higher during leftward than during rightward eye tracking.

On the basis of the pattern of MEP modulation during SP, we can then conclude that the observed changes in CSS excitability are compatible with a motor plan encoding an aiming movement of the hand toward the same target tracked by the eyes.

\section{Ocular and manual tracking systems are driven by a common command signal}

It is well known that when arm and eyes are simultaneously involved in a tracking task the performance of both systems considerably improves compared with when they move alone (Koken and Erkelens, 1992; Miall and Reckess, 2002). To account for these observations, a model has been proposed in which ocular and manual responses are controlled by completely independent sensorimotor systems, and performance improvement during combined tracking would result from an interchange of nonvisual signals between separate controllers (Gauthier et al., 1988; Lazzari et al., 1997; Scarchilli and Vercher, 1999). A place at which such exchange of information could occur is the cerebellum, where oculomotor signals would be used to exert a predictive control of manual tracking (Miall and Reckess, 2002). Our data do not exclude the existence of such mechanism in oculomanual coordination. However, the finding that a subthreshold motor plan for the arm is formed during SP, even when manual tracking is not required, is a strong argument in favor of the hypothesis that ocular and manual tracking systems are not completely independent but are driven by a common command signal and, therefore, share at least partially a common neural controller. This viewpoint is in agreement with the observation that gain adaptation imposed to the ocular pursuit influences also manual tracking responses (van Donkelaar et al., 1994), indicating that plastic changes must occur in a common neural substrate. Furthermore, the conclusion that the two systems share a common controller is also supported by the striking similarities in kinematics of eye and hand tracking movements, despite the considerable inertial differences of the two systems (Engel et al., 2000).
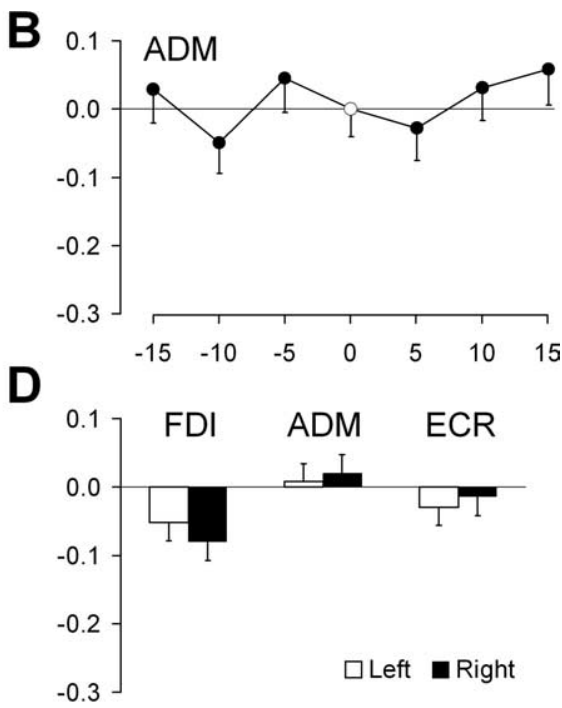

Eye position (degrees)

\section{Congruency of arm posture affects the degree of} eye-hand coupling

A main result of this study is that the excitability changes in the CSS, induced by SP eye movements, are strongly affected by the posture of the arm. Actually, a modulation of MEP amplitude during SP only occurs when the forearm is held in a pronated (palm down) posture (i.e., a posture that is normally associated with tracking, reaching, or pointing movements toward a visual object). In fact, the execution of these aiming tasks is more difficult, and for sure very unnatural, when performed with the hand held in a palm-up position. Interestingly, SP does not yield a significant modulation of CSS excitability when the forearm is passively held in supinated posture. It is then reasonable to conceive that the degree of eye-hand coupling during object tracking is adjusted on the basis of proprioceptive afferent signals, informing about the congruency of arm posture for the execution of a manual aiming movement.

This viewpoint finds support in recent data, showing that the pattern of excitability in the primary motor cortex can be modulated by proprioceptive afferent information about the actual position of the effector. For instance, the induced motion of index finger by TMS of the motor cortex turns from abduction to flexion, depending on the initial hand posture (Wassermann et al., 1998). In the same vein, it has been demonstrated that the excitability of the CSS controlling upper-limb muscles is enhanced when the arm is passively held in particular facilitatory configurations (Shimura and Kasai, 2002; Ginanneschi et al., 2005).

A modulatory effect of arm posture has also been described on the motor facilitation that accompanies the observation or imagination of actions (Fadiga et al., 1995, 2005; Izumi et al., 1995; Kasai et al., 1997; Hashimoto and Rothwell, 1999). Changes in corticospinal excitability show a high degree of specificity for the muscles that are actually involved in executing the observed (Fadiga et al., 1995; Strafella and Paus, 2000; Gangitano et al., 2001) or imagined (Yahagi and Kasai, 1998; Fadiga et al., 1999) movement. Similarly to our study, the pattern of motor facilitation while observing others' actions is deeply affected by the pronated/supinated posture of the forearm (Maeda et al., 2002; Urgesi et al., 2006). Moreover, MEP facilitation in hand muscles during imagery of finger move- 
ments is reduced when the imagined movement is incompatible with the subject's hand posture (Vargas et al., 2004).

One may argue that the changes in excitability of the motor cortex in this study could indeed be caused by mental imagery of a tracking arm movement by the subject. However, this explanation seems particularly unlikely, because we carefully avoided drawing subjects' attention to the possibility of making an aiming arm movement. Interviews after the experimental session confirmed the absolute absence of any imagery of manual tracking.

\section{Motor inhibition during eye tracking}

As already described, changes in MEP amplitude during SP cannot be simply ascribed to a subthreshold motor plan for manual tracking. In fact, the overall excitability of the hand CSS concomitantly shows a substantial decrease with respect to the level present during the fixation of a stationary target. This appears to be a generalized inhibition, because its amplitude is very similar in all investigated hand and wrist muscles (Fig. 2D). Nevertheless, it must be considered a very specific effect, because it occurs only when SP is performed with a pronated forearm (that is, in the posture in which MEP amplitude is also specifically modulated by the direction of eye tracking). No motor inhibition of the hand can instead be observed with a supinated forearm posture. The simultaneous occurrence of a generalized inhibition of the arm CSS and of excitability changes congruent with a motor plan for a hand movement is highly suggestive that, in the presence of a common drive to both eye and hand tracking systems, active inhibitory mechanisms are activated to switch off the arm motor system, whenever a manual tracking is not requested.

To our knowledge, a decrease in CSS excitability linked with the execution of eye movements has never been described before. Our study does not allow us to ascertain which part (cortical or spinal) of the CSS is involved in the observed modulation of excitability. Data in the literature suggest the possibility that this inhibition may at least partially occur at the spinal level. By testing the H-reflex in a finger flexor muscle, it has been shown that spinal excitability actually decreases during action observation (i.e., it is modulated in a way opposite to that found for the entire corticospinal pathway by means of TMS applied to the motor cortex) (Baldissera et al., 2001). Therefore, it is possible to speculate that a decrease of spinal excitability could be a mechanism to prevent muscle contraction in tasks that engage cortical motor systems but that do not require overt motor actions.

\section{References}

Baldissera F, Cavallari P, Craighero L, Fadiga L (2001) Modulation of spinal excitability during observation of hand actions in humans. Eur J Neurosci 13:190-194.

Bawa P, Chalmers GR, Jones KE, Søgaard K, Walsh ML (2000) Control of the wrist joint in humans. Eur J Appl Physiol 83:116-127.

Engel KC, Soechting JF (2003) Interactions between ocular motor and manual responses during two-dimensional tracking. Prog Brain Res 142:141-153.

Engel KC, Anderson JH, Soechting JF (2000) Similarity in the response of smooth pursuit and manual tracking to a change in the direction of target motion. J Neurophysiol 84:1149-1156.

Fadiga L, Fogassi L, Pavesi G, Rizzolatti G (1995) Motor facilitation during action observation: a magnetic stimulation study. J Neurophysiol 73:2608-2611.

Fadiga L, Buccino G, Craighero L, Fogassi L, Gallese V, Pavesi G (1999) Corticospinal excitability is specifically modulated by motor imagery: a magnetic stimulation study. Neuropsychologia 37:147-158.
Fadiga L, Craighero L, Olivier E (2005) Human motor cortex excitability during perception of others' action. Curr Opin Neurobiol 15:213-218.

Gangitano M, Mottaghy FM, Pascual-Leone A (2001) Phase-specific modulation of cortical motor output during movement observation. NeuroReport 12:1489-1492.

Gauthier GM, Vercher J-L, Mussa-Ivaldi F, Marchetti E (1988) Oculomanual tracking of visual targets: control learning, coordination control and coordination model. Exp Brain Res 73:127-137.

Ginanneschi F, Del Santo F, Dominici F, Gelli F, Mazzocchio R, Rossi A (2005) Changes in corticomotor excitability of hand muscles in relation to static shoulder positions. Exp Brain Res 161:374-382.

Hashimoto R, Rothwell JC (1999) Dynamic changes in corticospinal excitability during motor imagery. Exp Brain Res 125:75-81.

Izumi S, Findley TW, Ikai T, Andrews J, Daum M, Chino N (1995) Facilitatory effect of thinking about movement on motor-evoked potentials to transcranial magnetic stimulation of the brain. Am J Phys Med Rehabil 74:207-213.

Kasai T, Kawai S, Kawanishi M, Yahagi S (1997) Evidence of facilitation of motor evoked potentials (MEPs) induced by motor imagery. Brain Res 744:147-150.

Koken PW, Erkelens CJ (1992) Influences of hand movements on eye movements in tracking tasks in man. Exp Brain Res 88:652-664.

Lazzari S, Vercher JL, Buizza A (1997) Manuo-ocular coordination in target tracking. I. A model simulating human performance. Biol Cybern $77: 257-266$.

Maeda F, Kleiner-Fisman G, Pascual-Leone A (2002) Motor facilitation while observing hand actions: specificity of the effect and role of observer's orientation. J Neurophysiol 87:1329-1335.

Mather J, Lackner J (1980) Multiple sensory and motor cues enhance the accuracy of pursuit eye movements. Aviat Space Environ Med 51:856-859.

Miall RC, Reckess GZ (2002) The cerebellum and the timing of coordinated eye and hand tracking. Brain Cogn 48:212-226.

Mills KR, Nithi KA (1997) Corticomotor threshold to magnetic stimulation: normal values and repeatability. Muscle Nerve 20:570-576.

Neggers SF, Bekkering H (1999) Integration of visual and somatosensory target information in goal-directed eye and arm movements. Exp Brain Res 125:97-107.

Prablanc C, Echallier JF, Komilis E, Jeannerod M (1979) Optimal response of eye and hand motor systems in pointing at a visual target. Biol Cybern 35:113-124.

Scarchilli K, Vercher J-L (1999) The oculomanual coordination control center takes into account the mechanical properties of the arm. Exp Brain Res 124:42-52.

Shimura K, Kasai T (2002) Effects of proprioceptive muscular facilitation on the initiation of voluntary movement and motor evoked potentials in upper limb muscles. Hum Mov Sci 21:101-113.

Strafella AP, Paus T (2000) Modulation of cortical excitability during action observation: a transcranial magnetic stimulation study. NeuroReport 11:2289-2292.

Urgesi C, Candidi M, Fabbro F, Romani M, Aglioti SM (2006) Motor facilitation during action observation: topographic mapping of the target muscle and influence of the onlooker's posture. Eur J Neurosci 23:2511-2530.

van Donkelaar P, Staub J (2000) Eye-hand coordination to visual versus remembered targets. Exp Brain Res 133:414-418.

van Donkelaar P, Fisher C, Lee RG (1994) Adaptive modification of oculomotor pursuit influences manual tracking responses. NeuroReport 5:2233-2236.

Vargas CD, Olivier E, Craighero L, Fadiga L, Duhamel JR, Sirigu A (2004) The influence of hand posture on corticospinal excitability during motor imagery: a transcranial magnetic stimulation study. Cereb Cortex 14:1200-1206.

Vercher JL, Magenes G, Prablanc C, Gauthier GM (1994) Eye-head-hand coordination in pointing at visual targets: spatial and temporal analysis. Exp Brain Res 99:507-523.

Wassermann EM, Tormos JM, Pascual-Leone A (1998) Finger movements induced by transcranial magnetic stimulation change with hand posture but not with coil position. Hum Brain Mapp 6:390-393.

Yahagi S, Kasai T (1998) Facilitation of motor evoked potentials (MEPs) in first dorsal interosseous (FDI) muscle is dependent on different motor images. Electroencephalogr Clin Neurophysiol 109:409-417. 